\title{
Statik und Dynamik der beiden Phtalylchloride
}

\author{
Von \\ Wilhelm Csányi
}

Aus dem Chemischen Institut der Universität in Graz

(Mit 3 Textfiguren)

(Vorgelegt in der Sitzung am 28. November 1918)

Vorliegende Arbeit bildet die erste Mitteilung einer noch nicht abgeschlossenen Untersuchung über die Kondensation von Phtalylchlorid mit Phenolen. Die diesbezüglichen Studien haben vorläufig ergeben, daß Phtalsäureester, Phtaleine und Fluorane in mit den Versuchsbedingungen wechselnden Verhältnissen immer nebeneinander entstehen. Dies ließ es wünschenswert erscheinen, vornächst die Beziehungen, in welchen die beiden Phtalylchloride zueinander stehen, kennen zu lernen.

Nach einem jahrzehntelangen wissenschaftlichen Streite über die Struktur des Phtalylchlorids war es E. Ott ${ }^{1}$ gelungen, das bis dahin allein bekannte flüssige Chlorid $^{2}$ in sein Isomeres vom Schmelzpunkt $88.5^{\circ}$ überzuführen, welch letzteres die unsymmetrische Konstitution besitzt. Wie Ott angibt, ist das

1 Lieb. Ann., 392, (1912), 245, insbesondere $273 \mathrm{ff}$; interessant ist übrigens, daß schon im Jahre 1880 von E. v. Gerichten (Berl. Ber., 13, 417) die Ansicht ausgesprochen wurde, daß das Phtalyichlorid ein Gemenge von symmetrisch und unsymmetrisch geformtem Chlorid darstellt.

2 In Bei1stein's Handb., III. Aufl., (1896), Bd. 2, p. 1794, findet sich uber die physikalischen Konstanten des Phtalylchlorids folgende Notiz: flüssig, erstarrt bei $0^{\circ}$, Siedepunkt $275 \cdot 4^{\circ}$ bei $726 \mathrm{~mm}$. 
asymmetrische Chlorid bei gewöhnlicher Temperatur haltba:, wofern nur jede Berührung mit Chlorwasserstoffgas vermieden wird; bei Anwesenheit von Spuren dieser katalytisch wirkenden Substanz, durch mehrstündiges Erhitzen auf dem Wasserbade oder durch Destillation lagert es sich in das symmetrisch gebaute Isomere um. Die umgekehrte Umwandlung der symmetrischen in die asymmetrische Modifikation erfolgt über die Aluminiumchlorid-, beziehungsweise Zinnchloridverbindung, also durch Mediumwirkung.

Wenn man unter Tautomerie die Erscheinung einer intramolekularen, umkehrbaren Umlagerung versteht, bei welcher die Lage des Gleichgewichtes der beiden Isomeren innerhalb der weitesten Grenzen schwanken kann, so darf man schon aus Ott's Angaben auf das Bestehen von Tautomerie beim Phtalylchlorid schließen. Quantitativ aber ließen sich die hier obwaltenden Verhältnisse nur vom Standpunkt der Lehre vom chemischen Gleichgewicht und der Reaktionsgeschwindigkeit ermitteln.

Zunächst haben meine Versuche gezeigt, daß die Einstellung des Gleichgewichtes - bei Ausschluß von Katalyse und Mediumbeeinflussung - unterhalb des Schmelzpunktes des asymmetrischen Chlorids mit so geringer Geschwindigkeit erfolgt, daß die Aufnahme eines Zustandsdiagramms der beiden Formen durch anteilweisen Zusatz der einen Systemkomponente zur anderen möglich war. Einen Hinweis auf die relativ groBe Beständigkeit des asymmetrischen Chlorids, selbst noch bei der Schmelztemperatur, kann übrigens die zwischen 88 und $89^{\circ}$ scharf eintretende Verflüssigung abgeben; erst durch stärkeres Erwärmen oder Zufügen eines Katalysators werden die die Erreichung des Gleichgewichtszustandes hemmenden Momente beseitigt.

Das binäre Schmelzdiagramm (Fig. 1) zeigt die beiden Aste $A E$ und $B E$, die sich im scharf ausgeprägten Eutektikum $E$ schneiden; bei vorsichtigem ${ }^{1}$ Arbeiten lassen sie sich klaglos realisieren.

1 Vorsicht ist bier wegen der Zersetzungsgefahr durch Spuren von Wasserdampf anzuwenden; vgl. den experimentellen Teil der vorliegenden Mitteilung. 
Die Exstarrungserscheinungen können sich allgemein als abhängig erweisen:

1. von der Temperatur, auf welche die Schmelze erhitzt wurde,

2. von der Dauer dieser Erhitzung und

3. von der Art der Abkühlung.

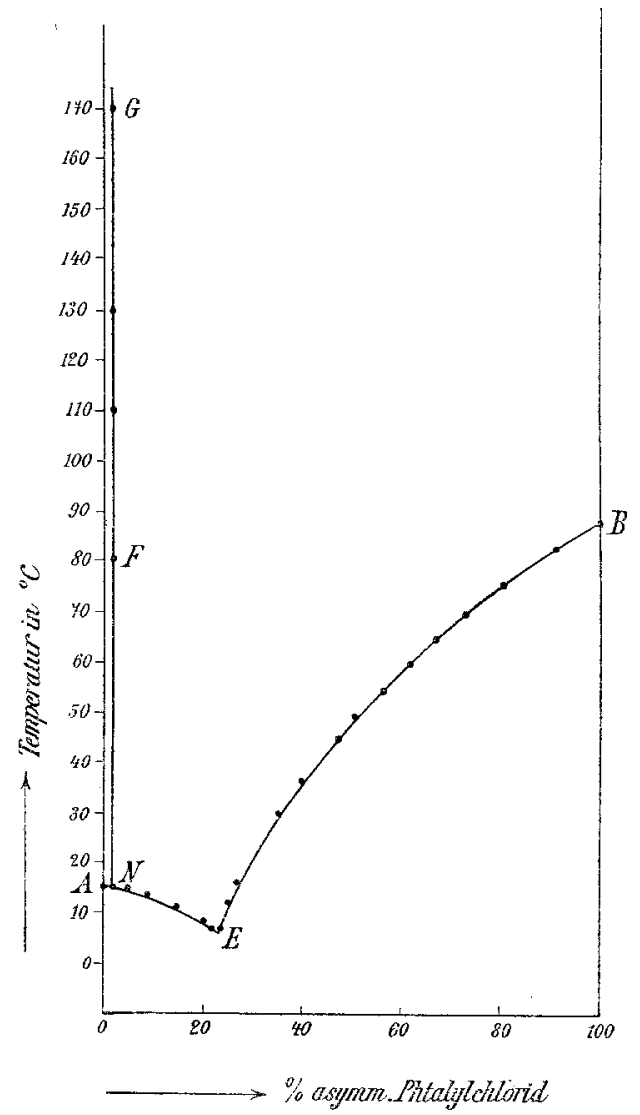

Fig. 1.

Was den gegebenen Fall anlangt, so ließ sich die Temperaturinvariabilität der ins Gleichgewicht gelangten Schmelze feststellen; in Fig. 1 bildet die Kurve $G N$ den graphischen Ausdruck für die Abhängigkeit der Zusammensetzung der Schmelze von der Temperatur - sie ist eine Gerade, die parallel zur Ordinate, beinahe mit dieser zusammenfällt. Durch 
Verlängerung der bei höheren Temperaturen ermittelten Kurve $G F$ ergibt sich ihr Schnittpunkt mit dem kurzen Ast der Schmelzkurve des Systems bei $N$ als einziger stabiler Tripelpunkt, welcher bekanntlich auch der "natürliche Schmelzpunkt genannt wird. Er wurde von beiden Seiten her übereinstimmend $\mathrm{zu}$ zirka $15^{\circ}$ gefunden; seine Lage auf dem Kurvenstück $A E$ wurde durch die Ermittlung der Tatsache eindeutig nachgewiesen, daß die Erstarrungspunkte von Schmelzen aus asymmetrischem Material nach längerem Erhitzen zunächst das eutektische Minimum passieren und dann wieder bis auf $15^{\circ}$ steigen, während die symmetrische Modifi-

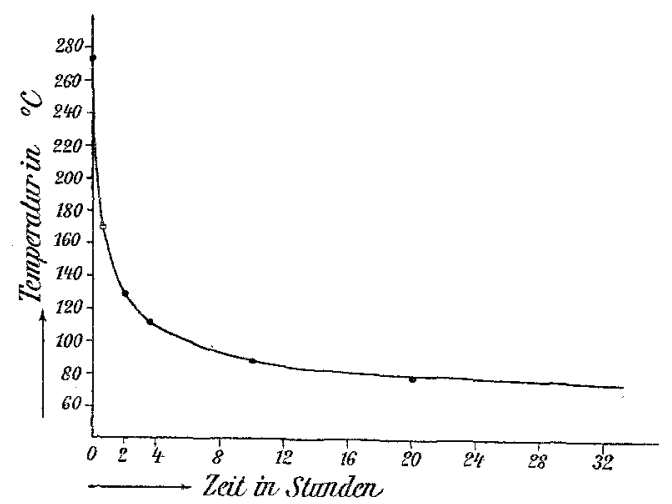

Fig. 2.

kation ihren Erstarrungspunkt auch nach beliebig langer Erhitzungsdauer annäherungsweise beibehält.

Es ist berechtigt, bei der praktisch völlig einseitigen Lage. des Gleichgewichtes von einem reinen, stabilen symmetrischen Phtalylchlorid und von der Umwandlung der instabilen asymmetrischen Form in die beständige symmetrische zu sprechen.

Mit der Temperaturinvariabilität des Gleichgewichtes im Schmelzfluß hängt es zusammen, daß - sowie letzteres einmal erreicht ist - die Dauer der Erhitzung wie auch die Art der Abkühlung die Lage des Erstarrungspunktes nicht mehr verändern.

Die Temperaturabhängigkeit der Umwandlungsgeschwindigkeit des asymmetrischen Chlorids in das symmetrische gibt Fig. 2 approximativ wieder. Beim Siedepunkt des Gleich- 
gewichtschlorides, das - wie erwähnt - fast reines symmetrisches Chlorid ist, geht die Umwandlung so gut wie momentan vor sich, bei der Temperatur des natürlichen Schmelzpunktes ist ihre Geschwindigkeit unmeßbar klein. Von einer genauen Bestimmung des Temperaturkoeffizienten mußte leider - wegen der Schwierigkeit der bezüglichen Untersuchungen Abstand genommen werden; aus der Kurve ist zu entnehmen, daß sein Wert für 10 Grade bei 2 oder darunter liegt; bei

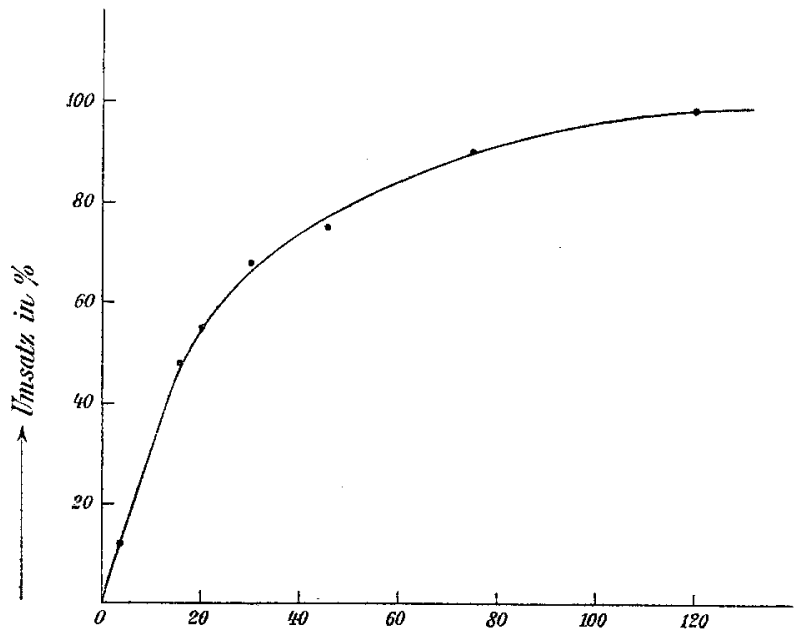

Zeit in Minuten

Fig. 3.

'Temperaturen oberhalb des Schmelzpunktes der höher schmelzenden Modifikation, von zirka $90^{\circ}$ bis $170^{\circ}$, beträgt er im Durchschnitt $1 \cdot 6$.

Die reine asymmetrische Modifikation lagert sich bei einer Temperatur von $130^{\circ}$ binnen 2 Stunden - also mit bequem meßbarer Geschwindigkeit - in die symmetrische um. Die Reaktion (vgl. Fig. 3) verläuft monomolekular. Die $K$-Werte, welche sich nach der Gleichung

$$
K=\frac{1}{t} \ln \frac{a}{a-x}
$$

berechnen, zeigen untereinander eine genügende Übereinstimmung; ihr Mittel beträgt 0.036 . 


\section{Experimenteller Teil.}

Bei der Darstellung des für vorliegende Untersuchungen benötigten asymmetrischen Phtalylchlorids wurde nach den Angaben von J. Scheiber ${ }^{1}$ vorgegangen, welche eine Verbesserung der Ott'schen Methode ${ }^{2}$ zur Reinerhaltung der Substanz bilden. Nach dem Fehlschlagen zweier Versuche, die mit käuflichem Aluminiumchlorid vorgenommen worden waren, wurde letzteres nach Gattermann ${ }^{3}$ hergestellt; bei Verwendung des auf diese Weise frisch bereiteten Materials gelang es denn auch, das asymmetrische Chlorid in sehr guter Rohausbeute $z u$ erhalten. Das nach Scheiber ${ }^{1}$ aus niedrig. siedendem Petroläther nach Verdampfen des Lösungsmittels zurückbleibende Produkt enthielt jedoch noch Phtalsäureanhydrid beigemengt; die vollständige Befreiung vom letzteren gelang - bei der großen Substanzmenge, die für die vorliegenden Untersuchungen nötig war - erst nach mühevollen Trennungen; es stellte sich heraus, daß es am zweckmäßigsten ist, dieselben folgendermaßen vorzunehmen:

Man unterbricht die Extraktion des Rohmaterials mit: Petroläther (bis $45^{\circ}$ siedender Anteil), wenn sich am Boden des Kolbens eine reichliche Menge der großen, derben Chloridkrystalle abgeschieden hat, filtriert sofort warm und erhält auf diese Weise das beinahe reine Material von der auch das Anhydrid enthaltenden Mutterlauge getrennt; das Lösungsmittel wird nun im Vakuum durch Verdunsten entfernt und der Rückstand, bestehend aus den Prismen des Chlorids und den feinen Nädelchen dẹ Anhydrids, von den letzteren durch Auslesen befreit; eine vollständige Trennung wird durch diese mechanische Methode nicht erreicht. Daher wird das noch verunreinigte Material wie das Rohchlorid nochmals der Extraktion mit niedrig siedendem Petroläther unterworfen; die sich nunmehr abscheidenden Prismen werden zusammen mit den eingangs durch fraktionierte Krystallisation erhaltenen fein pulverisiert und aus warmem Petroläther (zwischen $45^{\circ}$

1 Berl. Ber., 46 (1913), 2366.

Lieb. Ann., 392 (1912), 274.

3 "Praxis des organischen Chemilkers ", Verlag Veit, Leipzig. 
und $65^{\circ}$ siedender Anteil) umkrystallisiert; wird letztgenannte Behandlung zweimal vorgenommen, so ist man im Besitze einer reinen Substanz, welche nach dem Trocknen im Vakuum (über Paraffin und Schwefelsäure) einen scharfen Schmelzpunkt bei 88 bis $89^{\circ}$ zeigt.

Das symmetrische Chlorid kann durch Destillation des Handelsproduktes - wegen der nahe beieinander liegenden Siedepunkte - nicht völlig vom beigemengten Anhydrid befreit werden; durch Erwärmen mit der niedrig siedenden Petrolätherfraktion erhält man eine Lösung, welche beim Erkalten das Anhydrid ausscheidet; nach dem Verdampfen des Solvens im Vakuum hinterbleibt ein Produkt, welches im kühlen Raum $\left(+10\right.$ bis $12^{\circ}$ C. $)$ erstarrt. Man preßt auf einer porösen Tonplatte die flüssigen Anteile ab; nach einer Wiederholung der ganzen Operation zeigt die Substanz einen scharfen Schmelzpunkt bei 15 bis $16^{\circ}$, welcher der reinen symmetrischen Modifikation entspricht.

Die Methodik ${ }^{1}$ bei der Aufnahme von Tabelle 1, welche die Erstarrungspunkte in ihrer Abhängigkeit von der Zusammensetzung der Schmelze zeigt, war die folgende: Ein Glasrohr mit seitlich angebrachtem Tubus wurde mit einem doppelt durchbohrten Kork versehen, in welch letzterem Thermometer und Rührer so fest saßen, daß trotz der Rührmöglichkeit das Eindringen von Luftfeuchtigkeit tunlichst vermieden war; durch den Tubus wurde - nach vollständiger Trocknung des Rohres - soviel Substanz eingefüllt, daß nach dem Schmelzen die Thermometerkugel vollständig in die Flüssigkeit tauchte; dann wurde sogleich mittels Chlorcalciumaufsatzes geschlossen (das Säurechlorid setzt sich mit dem Wasserdampf der Luft merklich schnell in Phtalsäureanhydrid und Chlorwasserstoff um). Nach anteilweisem Zusatz abgewogener Mengen der anderen Systemkomponente wurde durch Aufschmelzen und langsames Abkühlen die Temperatur der primären Krystallisation be-

1 Vgl. R. Kremann, Monatsh. für Chemie, 25 (1904), 1229, und ds. threns, Samml. chem. und chem.-techn. Vorträge, 14 (1909), 213; ferner speziell über pseudobinäre Systeme: W. D. Bancroft, Journ. Phys. Chem., 2 (1898), $145 \mathrm{ff}$ - C. A. Soch, ibid. 2 (1898), 364. - F. C. Cameron, ibid. 2 (1898), 376 und 409 . 
stimmt, wobei die Zeitabkühlungskurve infolge Unterkühlung (die auch durch Zufügung von Keimen nicht ganz $\mathrm{zu}$ vermeiden war) stets das bekannte Unterkühlungsbild zeigte. Als wahre Erstarrungstemperatur wurde die dem ausgedehnten Horizontalteil der Kurve entsprechende angenommen.

Tabelle 1.

\begin{tabular}{|c|c|c|c|c|c|}
\hline Nr. & $\begin{array}{l}\text { Anfangs- } \\
\text { menge } \\
\text { asymmetr. } \\
\text { Chlorid }\end{array}$ & $\begin{array}{l}\text { Zusatz von } \\
\text { symmetr. } \\
\text { Chlorid }\end{array}$ & $\begin{array}{l}\text { Gesant- } \\
\text { menge }\end{array}$ & $\begin{array}{c}\text { Gewichts }= \\
\text { Molprozente } \\
\text { asymmetr. } \\
\text { Chlorid }\end{array}$ & $\begin{array}{c}\text { Temperatur } \\
\text { der } \\
\text { primären } \\
\text { Krystallisation }\end{array}$ \\
\hline 1 & $4 \cdot 50$ & 0.00 & $4 \cdot 50$ & $100 \cdot 0$ & $88 \cdot 5$ \\
\hline 2 & $4 \cdot 50$ & 0.43 & $4 \cdot 93$ & $91 \cdot 1$ & $82 \cdot 5$ \\
\hline 3 & $4 \cdot 50$ & 1.03 & $5 \cdot 53$ & $81 \cdot 4$ & $75 \cdot 5$ \\
\hline 4 & $4 \cdot 50$ & $1 \cdot 69$ & $6 \cdot 19$ & $72 \cdot 7$ & $69 \cdot 0$ \\
\hline 5 & $4 \cdot 50$ & $2 \cdot 26$ & $6 \cdot 76$ & $66 \cdot 6$ & $64 \cdot 5$ \\
\hline 6 & $4 \cdot 50$ & $2 \cdot 80$ & $7 \cdot 30$ & $61 \cdot 6$ & $60 \cdot 0$ \\
\hline 7 & $4 \cdot 50$ & $3 \cdot 46$ & $7 \cdot 96$ & $56 \cdot 5$ & $55 \cdot 3$ \\
\hline 8 & $4 \cdot 50$ & $4 \cdot 28$ & $8 \cdot 78$ & $51 \cdot 3$ & $49 \cdot 5$ \\
\hline 9 & $4 \cdot 50$ & $5 \cdot 01$ & $9 \cdot 51$ & $47 \cdot 3$ & $45 \cdot 0$ \\
\hline 10 & $4 \cdot 50$ & $5 \cdot 93$ & $10 \cdot 43$ & $43 \cdot 2$ & $41 \cdot 51$ \\
\hline 11 & $4 \cdot 50$ & 6.95 & $11 \cdot 45$ & $39 \cdot 3$ & 35.01 \\
\hline 12 & $2 \cdot 10$ & $3 \cdot 14$ & $5 \cdot 24$ & $40 \cdot 1$ & $37 \cdot 5$ \\
\hline 13 & $2 \cdot 10$ & 3.88 & $5 \cdot 98$ & $35 \cdot 1$ & $30 \cdot 5$ \\
\hline 14 & $1 \cdot 00$ & $2 \cdot 73$ & $3 \cdot 73$ & $26 \cdot 8$ & $16 \cdot 0$ \\
\hline
\end{tabular}

\begin{tabular}{|c|c|c|c|c|c|}
\hline $\mathrm{Nr}$. & $\begin{array}{l}\text { Anfangs- } \\
\text { menge } \\
\text { symmetr. } \\
\text { Chlorid }\end{array}$ & $\begin{array}{c}\text { Zusatz von } \\
\text { asymmetr. } \\
\text { Chlorid }\end{array}$ & $\begin{array}{c}\text { Gesamt- } \\
\text { menge }\end{array}$ & $\begin{array}{l}\text { Gewichts }= \\
\text { Molprozente } \\
\text { asymmetr. } \\
\text { Chlorid }\end{array}$ & $\begin{array}{c}\text { Temperatur } \\
\text { det } \\
\text { primären } \\
\text { Krystallisation }\end{array}$ \\
\hline 15 & 5.71 & 0.00 & $5 \cdot 71$ & $0 \cdot 0$ & $15 \cdot 5$ \\
\hline 16 & $5 \cdot 71$ & $0 \cdot 30$ & $6 \cdot 01$ & $5 \cdot 0$ & $14 \cdot 8$ \\
\hline 17 & $5 \cdot 71$ & $0 \cdot 60$ & $6 \cdot 31$ & $9 \cdot 5$ & $13 \cdot 3$ \\
\hline 18 & $5 \cdot 71$ & $1 \cdot 00$ & $6 \cdot 71$ & $14 \cdot 9$ & $11 \cdot \overline{5}$ \\
\hline 19 & $5 \cdot 71$ & $1 \cdot 47$ & $7 \cdot 18$ & $20 \cdot 5$ & $9 \cdot 5$ \\
\hline 20 & $5 \cdot 71$ & $1 \cdot 77$ & $7 \cdot 48$ & $23 \cdot 7$ & $8 \cdot 2$ \\
\hline 21 & $4 \cdot 83$ & $1 \cdot 40$ & $6 \cdot 23$ & $22 \cdot 5$ & $8 \cdot 5$ \\
\hline 22 & $4 \cdot 83$ & $1 \cdot 60$ & $6 \cdot 43$ & $24 \cdot 9$ & $12 \cdot 1$ \\
\hline
\end{tabular}


Zur Kontrolle der Temperaturablesungen wurde Schmelzenund Erstarrenlassen wiederholt; außerdem wurden Mischungen bestimmter Zusammensetzung öfters frisch bereitet, um fehlerhafte Werte auszuschließen, welche ihre Ursache in einer Zersetzung der Substanz hätten; eine solche konnte tatsächlich nach oftmaligem Eintragen von Zusätzen (Nr: 10 und 11 in Tabelle 1) trotz der früher angegebenen Vorsichtsmaßregeln beobachtet werden.

Die Einwagen wurden in einem Raum mit der Durchschnittstemperatur von $10^{\circ} \mathrm{C}$. vorgenommen; da diese unterhalb des Schmelzpunktes des symmetrischen Chlorids gelegen ist, konnte stets mit festem, krystallisiertem Material gearbeitet werden.

Nachdem das binäre Verhalten des Systems bei relativ niedrigen Temperaturen und innerhalb gewisser Zeitgrenzen erwiesen war, wurde die Abhängigkeit der Zusammensetzung der Schmelze von der Temperatur sowie die Lage des natürlichen Schmelzpunktes ermittelt (Tabelle 2).

'labelle 2.

\begin{tabular}{|r|r|r|r|r|}
\hline$A$ & $B$ & $C$ & $D$ & $E$ \\
\hline \hline 1 & 23 & $8 \cdot 0$ & 170 & $0 \cdot 5$ \\
2 & 100 & $88 \cdot 5$ & 170 & $0 \cdot 5$ \\
3 & 100 & $88 \cdot 5$ & 170 & $1 \cdot 0$ \\
4 & 0 & $15 \cdot 5$ & 170 & $0 \cdot 5$ \\
5 & 100 & $88 \cdot 5$ & 110 & 4 \\
6 & 100 & $88 \cdot 5$ & 80 & 20 \\
7 & 100 & $88 \cdot 5$ & 130 & 2 \\
& & & &
\end{tabular}

Es. bedeuten:

$A$ die Versuchsnummern,

$B$ die Zusammensetzung des Gemisches in Prozent an asymmetrischem Chlorid,

$C$ den Erstarrungspunkt nach dem kurzen Auftauen und sofortigem Abkühlen,

$D$ die Temperatur, auf welche nachher erhitzt wurde,

$E$ die Dauer des Erhitzens in Stunden. 
Nach dieser Erhitzungsdauer zeigten alle Schmelzen den natürlichen Erstarrungspunkt von $15^{\circ}$. Die mit reinem asymmetrischen Chlorid gemachten Versuche geben gleichzeitig die Erhitzungsdauer ungefähr an, die eben hinreicht, um die Umwandlung des asymmetrischen Chlorids in das symmetrische zu Ende zu führen. Die entsprechenden Werte sind im Diagramm 2 eingezeichnet.

Bei den Versuchen, bei denen längere Zeit auf hohe Temperatur erhitzt wurde, war die Methodik folgende: In ein durch regulierbare Flamme und Rührwerk auf konstanter Temperatur befindliches großes Ölbad wurde das mit der Substanz (stets zirka $2 g$ ) beschickte Gefäß so tief eingetaucht wie das Badthermometer; das Erhitzungsgefäß bestand aus einem mittelstarken Reagensrohr mit einem Aufsatz, der mit Natronkalk und.Chlorcalcium gefüilt war. Nach genügend langem Erhitzen wurde das Rohr möglichst rasch in ein zweites von etwas größerer Dimensionierung gebracht, das in Eiswasser stand; durch den Luftmantel sollte ein Springen des Gefäßes hintangehalten werden. Nachdem die Krystallisation der Substanz eingetreten war, wurde letztere aus dem Eiswasser entfernt und durch Handwärme zum Schmelzen gebracht; nun erfolgte auch der Austausch des. Chlorcalciumaufsatzes gegen den mit Thermometer und Rührer versehenen Korkverschluß; durch das »Abschrecken * der heißen Schmelze auf Eistemperatur war das »Hängenbleiben « des Gleichgewichtes bei der der Erhitzungstemperatur entsprechenden Zusammensetzung gewährleistet. Wie sich jedoch im Verlaufe der Versuche herausstellte, war infolge der Temperaturinvariabilität des Gleichgewichtes das rasche Sinkenlassen der Temperatur nicht notwendige Bedingung; nach langsamer Abkühlung der auf höhere Temperaturen erhitzten Schmelze wurde derselbe Erstarrungspunkt gefunden.

Der Verlauf von Versuch 7 (Tabelle 2) wurde zeitlich verfolgt; die erhaltenen Werte sind in Tabelle 3 wiedergegeben.

Der: Umsatz $x$ in Prozent (daher $a=100$ ) kann auf Grund des beobachteten Erstarrungspunktes dem Zustandsdiagramm Fig. 1 entnommen werden. 
Wie ersichtlich, durchwandern die Erstarrungspunkte bei der auf thren zeitlichen Verlauf untersuchten Umlagerungsreaktion das eutektische Minimum; dieser Úmstand bildet ein Kriterium für die Einseitigkeit des Gleichgewichtes, die ihren Ausdruck im praktischen Zusammenfallen des natürlichen Schmelzpunktes mit dem Schmelzpunkt des symmetrischen Chlorids findet.

Tabelle 3.

\begin{tabular}{|c|c|c|c|c|}
\hline Nr. & $\begin{array}{c}\text { Zeit } \\
\text { in Ninuten }\end{array}$ & $\begin{array}{c}\text { Erstarrungs } \\
\text { punkt } \\
\text { in Grad Celsius }\end{array}$ & $\begin{array}{c}\text { Unsatz } x \\
\text { in Prozent }\end{array}$ & $\frac{1}{t} \ln \frac{a}{a-x}$ \\
\hline 1 & 0 & 88.5 & 0 & - \\
2 & 4 & 80.0 & 12 & 0.0322 \\
3 & 16 & 51.0 & 48 & 0.0408 \\
4 & 20 & 43.0 & 55 & 0.0400 \\
5 & 30 & 25.0 & 68 & 0.6380 \\
6 & 45 & 8.0 & 77 & 0.0327 \\
7 & 75 & 13.0 & 90 & 0.0308 \\
8 & 120 & 15.0 & 99 & 0.0382 \\
& & & & \\
\hline
\end{tabular}

\section{Zusammenfassung.}

1. Das Phtalylchlorid kommt im festen sowohl als im flüssigen Zustande in zwei Modifikationen vor, welche zueinander im Verhältnis der Tautomerie stehen.

2. Das Gleichgewicht in der Schmelze ist einseitig aut Seite der tiefer schmelzenden Form gelegen; diese ist die stabile.

3. Das Gleichgewicht ist unabhängig von der Temperatur; daher muß der kalorische Effekt bei der Umwandlung der einen Modifikation in die andere sehr geringfügig sein.

4. Die Finstellung des Gleichgewichtes geht bei tiefen Temperaturen äußerst träge vor sich; mit dem Steigen der Temperatur wächst die Geschwindigkeit der Umwandlung in die stabile Form; beim Siedepunkt erfolgt die Umlagerung praktisch momentan. 
5. Der Temperaturkoeffizient der Umwandlungsgeschwindigkeit ist im Mittel $Q_{10}=1 \cdot 6$.

6. Die nach der Formel für monomolekulare Reaktionen berechnete und für die Temperatur von $130^{\circ} \mathrm{C}$. ermittelte Geschwindigkeitskonstante beträgt im Mittel 0.036 .

7. Der »natürliche Schmelzpunkt des Systems fällt praktisch mit dem Schmelzpunkt der tiefer schmelzenden Modifikation zusammen.

8. Das Eutektikum liegt bei $8^{\circ} \mathrm{C}$; das eutektische Gemisch enthält in 100 Teilen zirka 23 Teile der höher schmelzenden Form.

Meinem hochverehrten Vorstand, Herrn Prof. Dr. Anton Skrabal, sage ich auch an dieser Stelle für das mir stets bewiesene anregende und fördernde Wohlwollen meinen ergebensten Dank; Herrn Prof. Dr. R. Kremann bin ich für manch wertvollen Wink sehr verbunden. 